\title{
Subcutaneous Botulinum Toxin InJections for the Treatment of Chronically Painful Post-Craniotomy Scars: a Case Report of Two Patients Treated Successfully for More Than Eight Years
}

\author{
Dennis D. Dykstra, MD, PhD, Carlton Kimmerle, MD, Vasilios Kountis, DO, and Tanya Baxter, PT
}

Recently, the use of botulinum toxin type $A$ (BoNT-A) has been reported to help with neuropathic pain reduction in numerous conditions, including painful chronic scar formation. The mechanism by which BoNT-A inhibits pain is unclear, but it appears to involve a reduction of peripheral pain sensitization, which indirectly reduces central pain sensitization. Two patients who underwent surgery for brain tumor resection developed chronic painful neuropathic postcraniotomy scars. Their scar pain was not relieved with oral pain medications or trigger point injections with steroids but was helped significantly with BoNT-A injections. In the first patient, over the past 8 years, we have gradually increased her scar BoNT-A dose to 150 units diluted 2:1 with normal saline (NS) and injected the dose at 20 sites around her scar. She now reports her scar pain is $80 \%-90 \%$ resolved with the effect lasting 10 to 11 weeks. She denies side effects at this dose. In the second patient, over 9 years, we gradually increased the BoNT-A dose to 175 units diluted 2:1 with NS at 30 sites around her scar and in the left occipital area. She now notes her scar pain is $70 \%-75 \%$ improved from baseline with the effect lasting 8 to 10 weeks followed by a gradual reduction in benefit. She reports no side effects with this dose. The results of this 2-patient case study suggests BoNT-A (specifically onabotulinumtoxinA) may be helpful for patients with chronic postcraniotomy scar pain. Further studies on the dose, injection sites and timing follow-up injections are needed to determine treatment effectiveness for this patient population.

Key words: Scar pain, Botulinum toxin, chronic pain, subcutaneous, injections, post-craniotomy
Neuropathic pain is associated with an injury to the peripheral or central nervous system. Its presence can include hyperalgesia, allodynia, and hyperpathia. Opioids and analgesics are usually not helpful in the treatment of neuropathic pain patients. Other medications such as antidepressants and antiseizure medications are now used with better effect. Recently, the use of botulinum toxin type A (BoNT-A) has been reported to help with neuropathic pain reduction in

From : Department of Physical Medicine and Rehabilitation, University of Minnesota, Minneapolis, Minnesota

Author for correspondence: Dennis Dykstra, MD, PhD

Address: Department of PM\&R, MMC \#297, University of Minnesota, Minneapolis, MN 55455

E-mail: dykst001@umn.edu numerous conditions such as headaches, painful keloids, chronic neck pain after surgical dissection, chronic postthoracotomy scar pain, and trigeminal neuralgia (1-4).

The analgesic effects of BoNT-A were initially attributed to a decrease in muscle activity. However, further studies revealed that these analgesic effects occurred earlier and to a greater degree than the decrease in muscle activity, suggesting that BoNT- A may have a more complex mechanism of action on the pain system (5).

We present 2 cases of successful management of postcraniotomy neuropathic scar formation pain treated with BoNT- A injections. 


\section{CASE}

The first patient is a 43-year-old woman with a complex medical history that includes migraine headaches since age 15 and a more recent left oligodendroglioma diagnosis with subsequent resection, performed in 2004. Her headaches would occur on average 4 times per year without aura and include symptoms of nausea and photophobia. Postsurgically, she complained of ongoing left-sided head pain. She described the location of her postoperative head pain as focal, located over the surgical burr hole in the left parietal area. Prior to surgery, the patient had head pain in that generalized area; the pain level decreased somewhat after tumor resection. However, over the following month, the character of the pain changed from a pressure sensation to a more neuropathic pattern that she described as burning. The pain intensity was a constant 7 of 10 on the pain scale. Aggravating factors included change in weather patterns and cold temperatures. She was occasionally awakened from sleep due to pain. Pressing on the scar caused increased pain and triggered her migraine headache symptoms.

During the month after surgery, the patient also developed almost daily headaches and neck pain that lasted up to 23 hours per day. The headaches were similar to her previous baseline migraine headaches, only more intense and of longer duration.

To manage her new scar pain, we tried duloxetine, hydroxyzine, carbamazepine, morphine, and acetaminophen/oxycodone without significant success. We then injected her scar with one cc of $0.25 \%$ bupivacaine hydrochloride and $20 \mathrm{mg}$ triamcinolone. This provided about $35 \%-40 \%$ relief from scar pain, lasting for 2 weeks, but did not change her migraine headache pain. Because these therapies provided only limited reduction in her symptoms, we administered BoNT- A injections.

We initially utilized 25 units of BoNT- A (specifically onabotulinumtoxinA) diluted 2:1 with preservativefree normal saline (NS). The toxin was injected subcutaneously with a $30 \mathrm{G}$, one-inch needle and tuberculin syringe at 10 sites positioned equidistant around the burr hole and scar area. After 10 days, the patient reported a significant decrease $(65 \%)$ in scar pain, which lasted 6 weeks. Because the patient continued to experience migraine headache pain, under electromyographic guidance we injected the painful areas with 75 units of BoNT-A diluted 2:1 with NS. The areas injected were the procerus, right and left, corrugator, temporalis, and frontal muscles in the face and scalp (50 units) and the right and left midtrapezius muscles in her neck ( 25 units). The patient reported a $70 \%$ decrease in migraine headache pain, which lasted for 7 weeks.

Over the past 8 years we have gradually increased her scar BoNT-A dose to 150 units diluted 2:1 with NS and injected the dose at 20 sites (Fig. 1.). She now reports her scar pain is $80 \%-90 \%$ resolved with effect lasting 10 to 11 weeks. She denies side effects at this dose.

Also, over the past 8 years we have gradually increased her migraine headache pain dose to 50 units of BoNT-A diluted 2:1 with NS in her neck muscles (right and left trapezius, splenius capitis, and levators) and 100 units of BoNT-A diluted 2:1 in NS in her facial/scalp muscles. She currently reports that her migraine headache pain is reduced by $85 \%-90 \%$, her migraine headache days have declined to 2-3 per month, and her migraine headache duration is less than 6 hours per incident.

Overall, using this protocol, the patient's postsurgical scar and migraine headache pain has been reduced by $85 \%-90 \%$ with an 11 -week duration of effect.

The second patient is a 59-year-old woman with a history of left-sided head and postsurgical scar pain in addition to neck and shoulder girdle pain and tightness since she underwent a left acoustic neuroma resection on April 28, 2005. In addition to postsurgical pain, she had profound sensorineural hearing loss in her left ear postsurgically. The scar pain was most severe at the resection site, which the patient rated 8 of 10 on the pain scale. She described the pain quality as sharp/stabbing, burning, aching, throbbing, and pulling tightness of constant duration that radiated from the posterior portion of her left ear to the vertex of her head. Over a 3 year period she had trials of biofeedback, physical therapy, and acupuncture, and a pain management clinic managed her trigger point injections with steroid and anesthetic as well as C2 root blocks without relief. Oral anticonvulsant medications gabapentin, oxcarbazepine, and topiramate as 


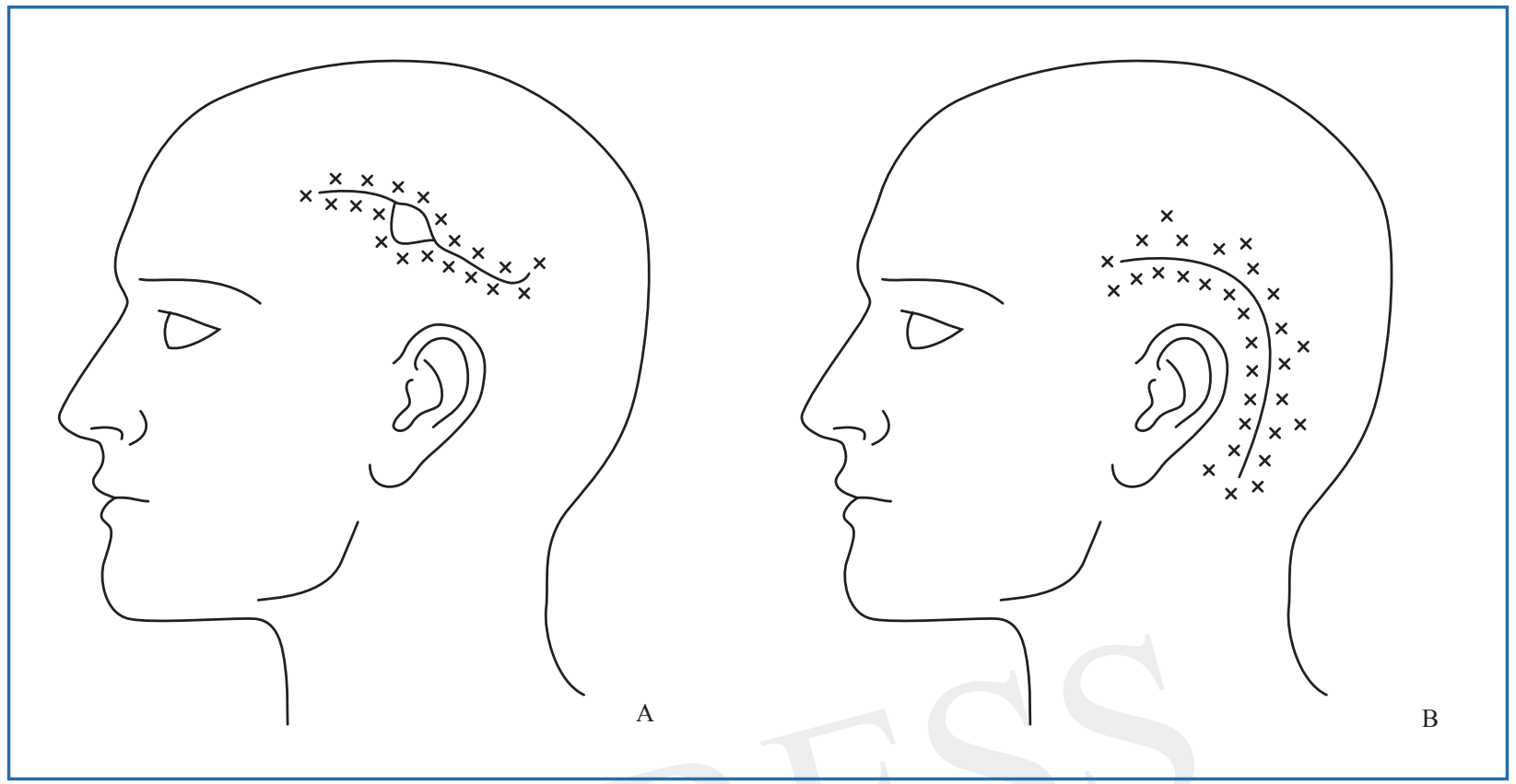

Fig. 1. Current Botulinum toxin type A injection sites for scar pain in: A. Patient 1 and B. Patient 2.

well as oral pain medications oxycodone, methadone, and fentanyl offered little pain relief and caused side effects of sedation and cognitive impairment.

Initial treatment to the left occipital area and postsurgical scar was with 50 units of BoNT-A diluted 2:1 with NS. The toxin was injected equally in 10 sites subcutaneously around the scar area with a 30G, one-inch needle and tuberculin syringe. After 10 days, the patient noted a significant decrease (about $50 \%$ ) in her pain for 5 weeks' duration. Over the next 9 years, we gradually increased the BoNT-A dose to 175 units diluted 2:1 with NS at 30 sites around her scar and in the left occipital area (Fig. 1.). She now notes her scar pain is 70\%-75\% improved from baseline with the effect lasting 8 to 10 weeks followed by a gradual reduction in benefit. She reports no side effects with this dose.

Because of the patient's neck and shoulder tightness and pain, under electromyographic guidance we also injected the bilateral rectus capitis, low splenius capitis, and lateral trapezius muscles with 50 units BoNT-A diluted 2:1 with NS. This offered the patient 4-5 weeks of muscle spasm and pain relief (a reduction from $7-8$ to 5 of 10 on the pain scale). Over the following 9 years we increased this dose to 200 units BoNT-A diluted 1:1 with NS. This provided the patient $75 \%$ pain and muscle spasm relief, compared to baseline, for 8 to 10 weeks.

Overall, her scar, head and neck, and shoulder girdle pain has improved $70 \%-75 \%$ with an 11 -week duration of effect.

\section{DISCUSSION}

The cause of scar tissue pain is thought to be secondary to skin nerve injury or nerve pressure from scar tissue formation. Sometimes a neuroma may form at a damaged nerve ending. Patients with scar tissue pain usually complain of burning, sharp, stabbing, shooting pain in the scar and surrounding area. Postoperative scars may trigger or increase headaches (6).

The mechanism by which BoNT-A inhibits pain is unclear. Animal model research observations suggest that under conditions of inflammation and sustained pain, BoNT-A toxin exerts its peripheral antinociceptive effects by inhibiting the release of pain mediators such as glutamate, substance-P, and calcitonin gene-related peptide (CGRP) and inhibition 
of vanilloid receptor activity. This direct reduction of peripheral pain sensitization has been found to indirectly reduce central pain sensitization associated with chronic pain (5).

In the first case study, the patient developed scar pain and increased migraine headaches about one month after craniotomy surgery. She was first treated for her scar and head pain with numerous medications, which did not help, and finally with a trigger point injection using an anesthetic and steroid. Her scar pain was improved somewhat with the trigger point injection, but her migraine headaches were not helped. Only after BoNT-A was injected into the scar and migraine headache pain areas did she receive significant relief in both pain areas.

In the second case study, the patient reported scar and temporal head pain near the resection site almost immediately after surgery. In addition, she developed

\section{REFERENCES}

1. Francisco GE, Tan H, Green M. Do botulinum toxins have a role in the management of neuropathic pain? A focused review. Am J Phys Med Rehabil 2012; 91:899-909.

2. Fabregat G, De Andrés J, Villanueva-Perez VL, Asensio-Samper, JM. Subcutaneous and perineural botulinum toxin type A for neuropathic pain: A descriptive review. Clin J Pain 2013; 29:1006-1012

3. Ranoux D, Attal N, Morain F, Bouhassira D. Botulinum toxin type A induces direct analgesic effects in chronic neuropathic pain. Ann Neurol 2008; 64:274-283.

4. Fabregat G, Asensio-Samper JM, Palmisani S, Villanueva-Per- neck and shoulder girdle pain and tightness. She was first treated for scar and head area pain with BoNT-A with good results. She was then treated with BoNT-A for her neck and shoulder pains, again with good results.

In conclusion, the medical literature includes numerous studies that suggest BoNT-A is helpful in treatment of patients with headache pain, musculoskeletal pain due to muscle spasticity, and (most recently) various chronic pain conditions with a neuropathic component $(1-3,7)$. The results of this 2-patient case study suggests BoNT-A (specifically onabotulinumtoxinA) may also be helpful for patients with chronic postcraniotomy scar pain.

Further studies on the dose, injection sites, and timing of follow-up injections are needed to determine treatment effectiveness for this patient population.

ez VL, De Andrés J. Subcutaneous botulinum toxin for chronic post-thoracotomy pain. Pain Pract 2013; 13:231-234.

5. Aoki KR, Francis J. Updates on the antinociceptive mechanism hypothesis of botulinum toxin A. Parkinsonism Relat Disord 2011; 17(suppl 1):S28-S33.

6. Ferreira Kdos S, Dach F, Speciali JG. Scar neuromas as triggers for headache after craniotomy: Clinical evidence. Arq Neuropsiquiatr 2012; 70:206-209.

7. Qerama E, Fuglsang-Frederiksen A, Jensen TS. The role of botulinum toxin in management of pain: An evidence-based review. Curr Opin Anaesthesiol 2010; 23:602-610. 